\title{
THE FEATURES OF INTERRUPTING, COLLABORATING, AND BACKCHANNELLING USED BY BROADCASTER AND CALLER IN TELEPHONE CONVERSATION
}

\author{
Elok Widiyati \\ Sultan Agung Islamic University \\ Semarang, Indonesia \\ elok_widiyati@yahoo.com
}

\begin{abstract}
Conversational analysis is a part of conversational structure that manages people's communication. It deals with pragmatics. Some of the conversational structures consist of features in interrupting, collaborating, and backchannelling (Celce-Murcia, 1995: 14). This research was intended to analyze how the three features were applied in the English conversation by broadcasters and callers in English Corner Program. This research was qualitative. The data consisted of eight conversations. The data were collected through the following steps: recording, transcribing, selecting, and reporting. Then, they were analyzed with conversational approach drawn on Celce Murcia (1995). It was revealed that the broadcasters and callers in the eight conversations used the features of interrupting, collaborating, and backchanneling. They did not fully perform common English expressions and gambits. However, they tended to perform the simpler and easier ones. This implied that the participants have not known the appropriate expressions. Based on the result of this research, it was concluded that the conversations in this study were less structured and patterned. It was suggested for the readers who learn about conversational structure, that they should pay attention not only to the linguistic components, but also the pragmatics in which the English conversation takes place, including participant, context, and topic of a conversation.
\end{abstract}

Keywords: Interrupting, collaborating, backchanneling, broadcasters and callers, English Corner Program.

\section{INTRODUCTION}

Information as well as technology is very important to facilitate people's interaction and communication in national, international, and intercultural situation. People can interact and communicate with others by different means such as speaking, writing, or gestures. The definition of language as given by Finocchiaro (1974:3) is that language is a system of arbitrary, vocal symbols, which permits all people in a given culture or other people who have learned the 
system of that culture to communicate or to interact. Thus, communication across language becomes even more essential.

Every language has four skills which have to be mastered by its learners. They are listening, speaking, reading, and writing. Listening and reading skills are grouped into receptive skills because learners only receive information from other sources. In the other hand, speaking and writing skills are grouped into productive skills because those make learners produce language to give information. That is why speaking is very important to be mastered comprehensively. It is fully considered that by practicing speaking regularly will make learners easily understand what actually language is.

So that producing spoken language by learners is the effective way in learning language. Many kinds of activities include speaking such as group discussion, panel discussion, daily conversation, debating, interview, asking for information, reporting, giving suggestion and advice, public speaking, small talk, and also taking part in English radio program directly, etc. Spoken language is an applicable skill related to broadcasting. Here learners can directly practice their competence by performing their ideas or thoughts.

\section{Discourse Analysis}

Before going to the further elaboration of discourse analysis, let us see what actually discourse is. Schiffrin (1988: 251) defines discourse as any unit of language beyond the sentence and conversation as any discourse which is produced by more than one person. Discourse includes both dialogic and monologic forms in either spoken or written modes. Conversation includes just spoken dialogue. We can say that the goal of discourse analysis is the systematic differentiation of a random list of sentences from text.

The study of discourse belongs to the study of language in use, which means that it is concerned not just with properties of linguistic factors that determine what messages are conveyed by the use of linguistic form and whether it counts as an acceptable contribution to the communicative enterprise. While the linguistic properties of an utterance may determine a range of possible interpretations, the actual message recovered by the hearer depends on its linguistic properties (Blackmore, 1988: 229). 
Social Interaction

Conversation is produced by more than one individual. Understanding its construction requires an examination of how efforts from different individuals are coordinated. The most basic type of coordination is between what the speaker means and what his addresses understand him to mean. All other types of coordination as in turn taking, choice of conversational topics, and course narration are really in service of the more basic coordination between meaning and understanding. Despite the fact that a general coordinative effort is required for all language use, the dependency of conversation on coordination has been explained not only in linguistic terms but also in terms of the nature of conversation as social interaction.

\section{The Nature of Communication}

The term "conversation" is used ambiguously in current literature, referring sometimes to any spoken encounter or interaction and sometimes more restrictedly, to talk occurring when a small number of participants come together and settle into what they perceive to be a few moments out off from instrumental tasks. A period of idling felt to be an end in itself during everyone is accorded the right to talk as well as to listen and without reference to a fixed schedule. Everyone is accorded the status of someone whose overall evaluation of the subject matter at hand is to be encouraged and treated with respect and there is no final agreement or synthesis is demanded, differences of opinion to be treated as unprejudicial to the continuing relationship of the parties. Canale (1983: 2) shows that communication is understood to have the following characteristics, it:

(1) is a form of social interaction therefore it is normally acquired and used in social interaction;

(2) involves a high degree of unpredictability and creativity in form and message;

(3) takes place in discourse and sosiocultural contexts which provide constraints on appropriate language use and also clues to correct interpretations of utterances;

(4) is carried out under limiting psychological and other conditions such as memory constraints, fatigue and distractions;

(5) always as a purpose (e.g. to establish social relations, to persuade, or to promise); 
(6) involves authentic as opposed to textbook-contrived language; and

(7) is judged as successful or not on the basis of actual outcomes.

In addition, communication is understood as the exchange and negotiation of information between at least two individuals through the use of verbal and non-verbal symbol, oral and written or visual modes, and also production and comprehension processes. In this sense, communication involves the continuous evaluation and negotiation of meaning on the part of the participants. Finally, it is assumed that authentic communication involves a reduction of uncertainty on behalf of the participants.

Communication is more than merely the exchange of information. When people take part in conversation, they bring to the conversational process shared assumptions and expectations about what conversation is, how conversation develops, and the sort of contribution they are each expected to make when people engage in conversation. They share common principles of conversation that lead them to interpret each other's utterances as contribution to conversation.

A conversation is quite different from many of speech events. It does not have specified setting, time, place, required roles other than persons, specified agenda, and a quorum of simply or more. Like other speech activities, however, conversations must be opened and commonly this is done though the use of an adjacency pair such a greeting-greeting, request-grant, question-answer, or statement-response.

\section{Communicative Competence and Actual Communication}

Canale (1983: 5) defines communicative competence as the underlying systems of knowledge and skill required for communication. Actual communication is the realization of such knowledge and skill under limiting psychological and environmental conditions such as memory and perceptual constraints, fatigue, nervousness, distractions, and interfering background noises.

Hymes (1972: 281) suggests that language and other forms of communication are relevant if:

(a) whether (and to what degree) something is formally possible; 
(b) whether (and to what degree) something is feasible in virtue of the means of implementation available;

(c) whether (and to what degree) something is appropriate (adequate, happy, successful) in relation to a context in which it is set and evaluated;

(d) whether (and to what degree) something is in fact done, actually performed, and what its doing entails

Grice (1975: 58) proposes that there are six different kinds of knowledge the participants in a language-event must possess, they are:

(1) Each of the participant must know his role and status

(2) The participants must know where they are in space and time

(3) The participants must be able to categorize the situation in terms of its degree of formality

(4) The participants must know what medium is appropriate to the situation

(5) The participants must know how to make their utterances appropriate to the subject matter and the importance of subject matter

(6) The participants must know how to make their utterance appropriate to the domain to which the situation belongs.

Canale (1983: 6) perceives that communicative competence refers to knowledge and skill in using this competence when interacting in actual communication. Knowledge here refers to what one knows (consciously or not) about the language and about other aspects of communicative language use. Skill here refers to how well one can perform this knowledge in actual communication.

Competence has been related conceptually to the ability to discriminate between well-formed and deviant sentences. The application of the criterion of well-formedness has never been unproblematic and developments in transformational or generative theory have tended to make its application more difficult rather than less so (Coulthard, 1992: 61).

Components of Communicative Competence

Celce et.al. (1995: 10) proposes model or components of communicative competence. They represent their model of communicative competence as a 
pyramid enclosing a circle and surrounded by another circle (see Figure 1 below).

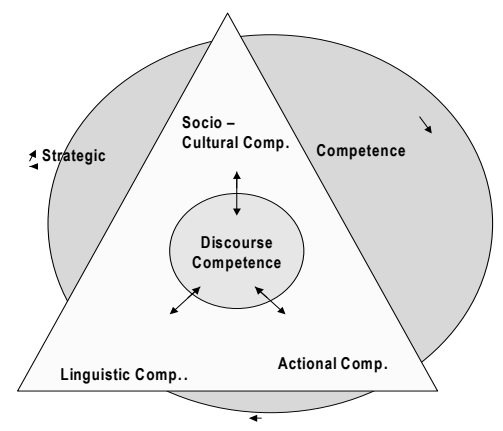

Figure 1: The model of Communicative Competence by Celce, et.al. (1995: 10)

The circle within the pyramid is discourse competence. The three points of the triangle are sociocultural competence, linguistic competence, and actional competence. They place the discourse component in a position where the lexicon-grammatical building locks, the actional organizing skills of communicative intent, and the sociocultural context come together and shape the discourse and each of the other three components. The circle surrounding by the pyramid represents strategic competence, an ever-present and potentially unable inventory of skills that allows a strategically competent speaker to negotiate messages and resolve problems or to compensate for deficiencies in any of the other underlying competencies.

\section{Discourse Competence}

Canale et.al. (1983: 5) defines discourse competence as the ability to combine language structures into different types of cohesive texts (e.g., political speech and poetry). Then they elaborate that discourse competence concerns mastery of how to combine grammatical forms and meaning to achieve a unified spoken or written text in different genres. Unity of a text is achieved through cohesion in form and coherence in meaning. Cohesion deals with how utterances are linked structurally and facilities interpretation of a text. Coherence refers to the relationship among the different meaning in text where these meaning may be literal meanings, communicative functions, and attitudes. 
Celce, et.al. (1995: 13) explain that discourse competence concerns the selection, sequence, and arrangement of words, structures, sentences, and utterances to achieve a unified spoken or written text. This is where the bottomup lexico-grammatical micro level interacts with the top-down signals of the macro level of communicative intent and socio cultural context to express attitudes and messages and to create texts.

Celce, et.al. (1995:14) explain components of conversational structure. They include:

\section{Interrupting}

Interrupting people is an important skill in any language. It is sometimes difficult to do in another culture where the gestures or ways of speaking are very different from our own. As always, the formality of the situation and the relationship of the speakers will affect the way people talk to each other. Giving interruption to others can use some expressions below:

(1) Asking for, giving and not giving permission

(2) Making and agreeing to request.

Speakers need to try predicting questions will come out. In order to do that, they have to do repetition in sentences they do not fully understand, so the interlocutor repeats it. And they have to say 'Huh', 'Pardon me', 'Excuse me', or I didn't understand." There is no anything can stop the chance to speak faster than make a silence or saying 'Yes'. Gambits attracting interlocutor's intention consist of opening and interruption, for instance: 'Sorry, but......", "Excuse me for interrupting, but.....", "I might add here....", "I'd like to comment on that....", "May I add something....", "May I say something here....", "I'd like to say something....", "May I ask a question...."

Most people do not mind interruptions if they are short ones. There is something we can do if the interruption is not a quick one and if it is not an emergency. In conversation, it is considered impolite to interrupt in the middle of a sentence. We should wait until the sentence has been complete. This is called turn taking and helps minimize the confusion that comes from not listening to what the other person is saying. Interrupting strangers is normally not done since it is not considered polite to eavesdrop or listen in on someone else's conversation. However, it sometimes happens on occasions when someone is being helpful. In an elevator, for example, if you hear people discussing which 
floor they should get off on and they have the wrong one, you can interrupt to give them the correct information (Tillitt and Bruder, 1985: 78).

\section{Collaborating and Backchanneling}

Backchannel is a term from conversational analysis and discourse analysis. This refers to the verbal and non-verbal feedback which a listener gives to a speaker during an interaction (for example, 'Yes', 'Mmm' or 'I see'). Backchannel or engage is realized by 'Mm', 'Yeah'. Its function is to provide minimal feedback while a speaker is not interrupting the flow of the other participant's utterance (Coulthard, 1992: 133).

Back-channel 'Uhhuh' from a potential next speaker allows a current speaker to continue talking. Thus, since a continuation may be contingent on the placement of another's verbalization, it is not defined merely as monologue. In short, even if it is not openly designed for a recipient, whatever occurs in the presence of another is potentially communicative and potentially functional in the achievement of coordinated talk.

\section{METHODS}

\section{Research Design}

Conversational analysis is inductive. It is a study of recurring patterns across many records of naturally occurring conversation (Levinson, 1983: 287). Patterns of conversation include the procedure and expectation. They are conceived of and employed by participants themselves in producing and understanding conversation.

Conversational analysis is an empirical research. The research is based on observations in the media in which I got the data. In this research, I observed the conversational structure used by broadcasters and callers in English Corner Program.

The data of this research consisted of eight recorded conversations. The research took place in radio of English Corner Program. The subjects in this study were the caller and the broadcaster having a phone conversation and delivering the preference inside. 
Procedures of Data Collection

The first step in collecting data was recording. I recorded to get the data in the form of spoken language. I listened and transcribed the conversation. And then I played the records repeatedly to ensure that there were no words skipped. I identified the sentences and categorized them into the preference structure. The procedures of collecting data are divided into several steps: recording, transcribing, selecting, and reporting.

Data Analysis

In the core of analysis, I applied the correlations of content and format in adjacency pair by Levinson (1983). They consist of first part and second part. Moreover, the second part is categorized as preferred and dispreferred.

I described the recorded data of the conversations between the broadcaster and caller. Here, I analyzed the data by describing how the preference structure occurred. After describing and interpreting all the data, I discussed the problems in this study.

\section{FINDINGS}

\section{Interrupting}

Speakers usually cannot avoid delivering interruption in a conversation. It is because of the intention they want to emphasize a certain point in their speaking. Meanwhile, limited time affects them to do it in such away in order to make the utterance delivered stays in their mind and delivered well.

There are three kinds of interruptions delivered by the broadcasters and the callers. They were making a request, agreeing to request and asking for permission. The examples of making a request are "Hey, don't make many laughs yeah", "OK overtime yeah". Those two expressions delivered in order to the interlocutor did something. The first example made the interlocutor did not produce many laughs while speaking and the second example aimed as signal that the time for calling the program was nearly over.

"It's OK" is one example of agreeing to request. This is very simple expression delivered by the broadcaster when she was asked to do something. In asking for permission the caller used polite expression that was "OK. By the 
way, may I ask you a question?" This polite expression showed that the interruption done by the caller was patterned based on interruption gambit.

Some adjacency pairs expressed interruption used by the broadcasters and the callers are:

(1) C : Specially 'met'.

B : // Good not 'met' Yeah. It's OK.

C produced Indonesian term in adjacency pairs (1) so B suddenly made correction to what C said. B translated the word 'met' into 'good'. Expression of 'It's OK' delivered by $\mathrm{B}$ tended to give permission for $\mathrm{C}$ to say something. $\mathrm{B}$ expected that $\mathrm{C}$ would not say 'met' anymore. And $\mathrm{C}$ was successful in understanding that simple expression. $\mathrm{C}$ directly gave a response of an expected answer but she still used that word again.

Denial is the preferred response to blame which reflects the tendency to read 'silence' as a dispreferred second, i.e. as an admission of guilt. However, sometimes there are certainly situations in which a silent response to an accusation rules the accuser out of order.

(2) B : Hey, don't make many laughs yeah because you will cry later if you always laugh, laugh, and laugh.

C : Yes

B delivered a prohibition to $\mathrm{C}$ implicitly in adjacency pairs (2). This part can be regarded as interruption because it aims to stop $\mathrm{C}$ laughing in order to make the conversation runs well. $\mathrm{C}$ gave a response verbally and stopped laughing directly. There should be a permission to do interruption because the gambit about it also emphasizes in delivering appropriate and polite interruption by saying "Excuse me" or "Sorry". But in the other hand, many speakers ignore about this one. Interruption produced by speakers when one of them fells that the previous utterance given by the interlocutor does not meet with the prospective talk he wants to deliver later. So abruptly he cut the talk indicating that he produces an interruption.

(3) $\mathrm{C}:$ // Celebrate e........Idul Fitri day with all my family I mean. 
B : OK. Overtime yeah.

Interruption produced by B in adjacency pairs (3) because B thought that $\mathrm{C}$ had to finish his talk because of the limited time. B said "overtime" meant that he did not give more time for $\mathrm{C}$ to continue the talk. But unfortunately the way B interrupted was not polite. It should have been initiated by "Excuse me" or "Sorry to interrupt".

(4) C : OK. By the way, may I ask you a question? B : Yes, please.

Interruption in adjacency pairs (4) indicated that the speaker attracted the interlocutor to pay attention with something he wanted to ask. The second preferred pair part "Yes, please." indicated that the speaker gave permission to the interlocutor to continue his speech. The expression used to interrupt in this conversation was "By the way, may I ask you a question" either was not preceded by "I'm sorry" which could be more polite as interruption gambit should be.

Generally we need to ask for someone's attention before speaking. So, making interruption in a conversation needs permission whether the interlocutor let us interrupt him or not. The expressions usually used in getting someone's attention are "Excuse Me" or "Sorry" (Zwier, 2003: 2).

Interruption happens when the second speaker knows the intention of the interlocutor by saying what he knows that the next words are in her mind.

(5) $\mathrm{C}$ : My mom used to....

B1: // Turn on the lamp.

Interruption happened in adjacency pairs (5). However both speakers did not use any gambit expressing interruption such as "Excuse me" or "Sorry". I can say that sometimes interruption done by other is very useful in retrieving the information had to say before. In association with the turn taking system, where a special set of procedures operates to reduce and resolve overlap, this should arise despite the rules assigning turns. But there are overlaps allowed 
(and thus their location and nature predicted) by the rules, and overlaps that contravene the rules (interruption).

(6) $\mathrm{C}$ : Like the e....

B1 : But you didn't fear with speaking in front of this.

The interruption produced by B1 in adjacency pairs (6) was very abrupt. B1 should have given apologizing expression before interrupting C's unfinished statement. It seemed that $\mathrm{C}$ was not ready to deliver her statement because she made a silence among. This part was a transition between statement and denial produced by different speakers. These two pair parts above are categorized as inappropriate one.

Interruption delivered when a speaker wants to say the same or different thing from the previous speaker. However, the point in interruption is that the speaker emphasizes something he does not want to say. Interruption does not only occur when a speaker produces backchannel in the middle of the talk. However it also occurs when he wants to change the talk.

\section{Collaborating and Backchanneling}

Collaborating and backchannelling have an important role for the speaker and the interlocutor. Both of them give feedback each other by giving reinforcement. The reinforcement here can be performed by backchannel itself. An interlocutor produces 'Mm' while a speaker speaking. This minimal utterances indirectly gives a motivation for the speaker to continue his talk.

Backchannel functions as feedback produced by listeners to speakers in showing intention to the utterance delivered while speaker speaking. Most of the backchannels used by the broadcasters and the caller were "Yes" and "Mm". Sometimes they applied them to keep the continuation of talk and to minimize the interruption. Listeners also produced longer backchannels as "OK. Mm. Yup". It shows that the listener let the speaker keep talking buy giving high reinforcement in the form of longer backchannel.

Some adjacency pair parts expressed collaborating and backchanneling used by the broadcasters and the callers were:

(7) $\mathrm{C}:$ In UMK 
B : Yes

The backchannel produced by $\mathrm{B}$ in adjacency pairs (7) was a part of collaboration in the topic itself. It was realized by 'Yes'. It did not realize any element of move structure. Here it functioned to justify the information delivered from the interlocutor.

Backchannels which are frequently uttered in the conversation usually has a problem dealing with motivation decrease. It is not a surprise when some speakers usually forget about what will they say later on because backchannels delivered by their interlocutor. However, it will not happen when they manage the conversation well.

(8) B : Oh Geng telenovela. My gosh! Still there telenovela for today? No, I think. There's no telenovela program on television yeah.

C : // O yes

A protest was delivered by B in adjacency pairs (8). Its function was to raise an objection to a preceding utterance. It acknowledged the utterance while disputing its appropriateness while C accepted B's protest by producing backchannel.

(9) C : Mm.. OK today perhaps I want to

B : Mm..Yeah

B produced backchannel in adjacency pairs (9) by delivering feedback to $\mathrm{C}$ during the interaction and also allowed $\mathrm{C}$ to continue talking. When $\mathrm{C}$ requested a song in actually $\mathrm{C}$ did not find what the title was. So $\mathrm{C}$ just mentioned the singer.

Topical coherence is simple point cannot be perceived as residing in some independently calculable procedures for ascertaining, for example shared references across utterances. Rather, topical coherence is something constructed across turns by the collaboration of participants.

(10) C : OK. Time is running up. So I just wanna say hey 
B $\quad:$ // OK mm yup

Backchannel produced by B in adjacency pairs (10) indicated that he let $\mathrm{C}$ say hello to friends. Without taking the time $\mathrm{C}$ directly took the next turn to send regards to other listeners. Backchannels produced by speaker are mostly "mm' and "yeah". These forms function as the attention to the interlocutor while he is speaking. It seems that the backchannel producers really know and understand about what is talking about at that time.

(11) B1 : // Eh sorry yahoo.co.id.

B2 : Mm

Backchannel given by B2 in adjacency pairs (11) showed that the correction delivered before was correct. This part was inconsistent because the previous part was preclosing in the radio telephone conversation context. It looked like that all the speakers did not finish the conversation directly after they did preclosings. They could not control their conversation whether they had to finish or not because they prolonged the conversation. It seemed that the closing needed longer time.

(12) C : / / I can say that actually phobia is when we afraid about something illogically. There is a reason who make someone afraid about something.

B2: $\mathrm{Mm}$

Backchannel delivered by B2 in adjacency pairs (12) let the speaker continued his utterance without being interrupted. In this case, backchannel delivered in the middle of the conversation when the speaker gave different arguments. It means that the backchannel functioned as reinforcement to $\mathrm{C}$ in giving new argument. 


\section{CONCLUSION}

To conclude, the eight conversations investigated were structured in line with the principles of conversational analysis. The conversation between the broadcasters and the callers constituted natural conversation. And they applied the patterns of conversational structure. The features of interrupting, collaborating, and backchannelling were produced by the participants. The occurrences of overlaps and gaps did not have effect to transition on turn taking in general.

I hope this article can enrich the broadcasters' and the callers' knowledge on the importance of the conversational structure. It also helps them to understand and practice good conversation. The students would be able to enrich their knowledge about the conversational structure of English conversation.

\section{REFERENCES}

Blakemore, D. 1988. The organization of discourse. In Newmeyer. Pp.229-49.

Canale, M. 1983. From communicative competence to communicative language pedagogy. In Richard,J.C and R.W.Schmidt. Pp.2-27.

Celce, M. et.al. 1995. Communicative Competence: A Pedagogically Motivated Model with Content Specifications. IAL,6/2. Pp.5-35.

Cole, P and J.Morgan (e.d). 1975. Syntax and Semantics 3: Speech Act. New York: Academic Press.

Coulthard, M(e.d.). 1992. Advances in Spoken Discourse Analysis. London: Routledge.

Finocchiaro, M. 1974. English as a Second Language: from Theory to Practice. New York: Regents Publishing Inc.

Grice, H.P. 1975. Logic and conversation. In P. Cole and J. Morgan. P.p. 43-58.

Hymes, D. 1972. On communication competence. In J.B. Pride and J. Holmes. P.p. 269-93.

Levinson, S.C. 1983. Pragmatics. Cambridge: Cambridge University Press.

Newmeyer (e.d.). 1988. Linguistics: The Cambridge Survey: Volume IV.Language:

The Socio-cultural Context. Cambridge: Cambridge University Press. 
Pride, J.B and J. Holmes. 1972. Sociolinguistics, Selected Readings. Harmondsworth: Penguin.

Richard, J.C and R.W. Schimdt (e.d.). 1983. Language and Communication. London: Longman.

Schiffrin, D. 1988. Conversation analysis. In Newmeyer. Pp.251-73.

Tillitt, B. and M.N. Bruder. 1985. Speaking Naturally. Cambridge. Cambridge University Press.

Zwier, L. J and A. Hughes. 2003. Essential Functions for Conversation. Hongkong: Asia Pacific Press Holdings Ltd. 